\title{
Actin levels fluctuate in the seminiferous epithelium at various stages during spermatogenesis in the rat
}

\author{
Cristian ACOSTA, Mabel FósCOlO, Juan Carlos CAViCCHIA* \\ ${ }^{1}$ Instituto de Histología y Embriología de Mendoza (IHEM)-CONICET, Universidad Nacional de Cuyo, 5500, Mendoza, Argentina
}

Key words: Beta actin, Seminiferous epithelium, Trans-illumination assisted microdissection

\begin{abstract}
The testis is a double gland comprised of the sperm-producing seminiferous tubules and a complex endocrine interstice. The former structures generate and release whole cells (exocrine aspect of the gland) while the latter synthesize and release androgens and related hormones. The testis also has poorly understood paracrine connexions. These connexions play an important role during spermatogenesis. A key molecule within these structures is $\beta$-actin. Therefore, the present study aims at examining the expression pattern of $\beta$-actin during the various stages of the spermatic cycle in the rat. To achieve this goal, we used a combination of trans-illumination assisted microdissection of seminiferous tubules, confocal immunofluorescence and Western blot analysis. Our experiments revealed that the levels of $\beta$-actin fluctuate significantly during spermatogenesis, peaking immediately after spermiation.
\end{abstract}

Earlier studies established the morphological and functional bases of the seminiferous epithelium, the sustentacular Sertoli cell and the Sertoli-Sertoli gap junctions that, alongside with the $\beta$-actin cytoskeleton, make up the hemato-testicular barrier (see e.g. Dym and Cavicchia, 1977; Russell et al., 1989a). Russell demonstrated the determinant role of $\beta$-actin and other cytoskeleton components in the shaping, migration and release of the spermatid towards the excretory pathway (Russell et al., 1989b). Others have also highlighted the importance of $\beta$ - and F-actin during spermatogenesis (Kumar et al., 2016; Tang et al., 2016; Li et al., 2017). One essential feature of the seminiferous tubule is its intimate coordination with the interstice: each functional cycle involves drastic morpho-functional changes in their interplay (Losinno et al., 2012). However, a detailed examination of the $\beta$-actin levels and expression pattern at various stages throughout the seminiferous tubule was lacking.

In order to shed some light on this important question, we used the trans-illumination assisted microdissection method described by Parvinen (1993). This technique allowed us to identify different functional segments by recognizing specific morphological features of the seminiferous epithelium that can be seen using a stereomicroscope. We classed the seminiferous tubule into 5 main segments (in parenthesis, Parvinen's equivalent staging): I (Stage I), II (Stages II to VI), III (Stages VII and VIII), IV (Stages IX to

\footnotetext{
* Address correspondence to: Juan Carlos Cavicchia, jccavic@fcm.uncu.edu.ar

This paper belongs to the 60th Anniversary Collection of the Instituto de Histología y Embriología de Mendoza (IHEM)
}

XII) and V (Stages XIII and XIV). Although our classification is arbitrary each segment represents separate, main events, during spermatogenesis (Parvinen and Vanha-Pertula, 1972). The present study combines double-fluorescence immunohistochemistry, confocal microscopy and western blot analysis to examine the changes in the levels and pattern of expression of $\beta$-actin at various stages during the spermatogenic cycle. Segments of seminiferous tubules classed as mentioned above were harvested from 6 male adult Wistar rats (250-300 g body mass). Half the tissue was fixed for $1 \mathrm{~h}$ in Zamboni's fixative and then embedded in paraffin following standard procedures (Acosta et al., 2014). Tissue was cut in serial sections of $5 \mu \mathrm{m}$ and double immunofluorescence against $\beta$-actin (1:200, AC-15, Santa Cruz Biotechnology, sc-69879, RRID: AB_1119529) and DAPI was performed (Haskins et al., 2017). Images were captured in an Olympus FV-1000 confocal microscope. The other half of the tissue was immediately submerged in Laemmli's protein extraction buffer supplemented with a proteinase and phosphatase inhibitor cocktail (Halt, Thermofisher). Western blotting was carried out using PVDF membranes and denaturing conditions as previously published (Acosta et al., 2014). Quantitation was performed using ImageJ as described elsewhere (Benitez et al., 2017).

Fig. 1 shows that $\beta$-actin levels (in green) increase from segments I through IV, leveling down at segment V. Its distribution also changes: from being diffuse at segments I and II to be located in close proximity to the tubule's lumen at segments III and IV, albeit the latter also exhibits a substantial increment in the absolute level of the protein. This 
pattern in segment IV indicates abundance of intercellular junctions between spermatids close to spermiation. Scale bar represents $100 \mu \mathrm{m}$.
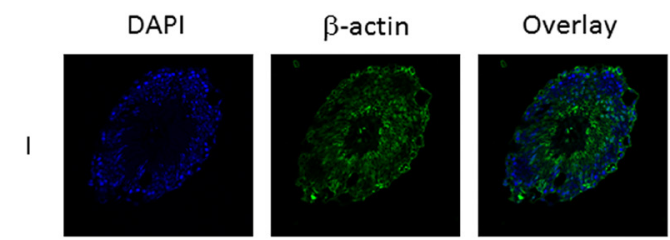

II
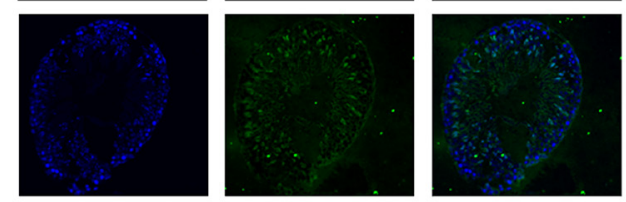

III
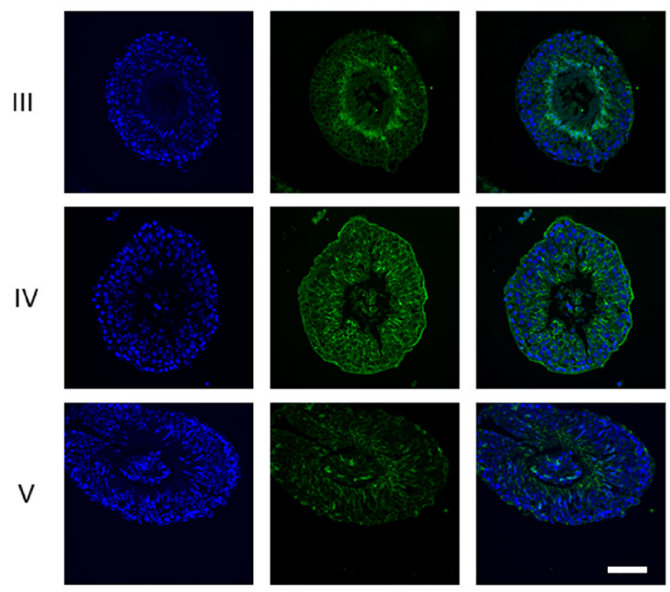

FIGURE 1. Representative confocal images of transverse sections of seminiferous tubules immunostained against $\beta$-actin (in green) and DAPI (in blue). Roman numbering indicates the five segments used in this study (see main text for details). Merged images depict the successive waves of changes in the expression of $\beta$-actin during spermatogenesis, which peaks at stage IV. Scale bar: $100 \mu \mathrm{m}$.

A

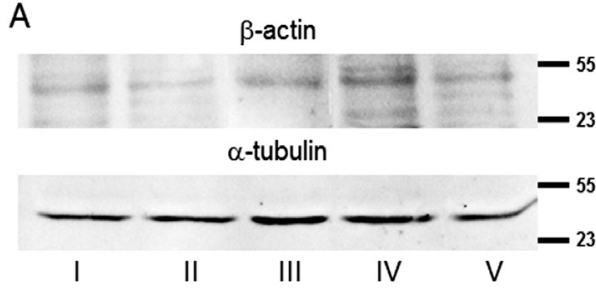

B

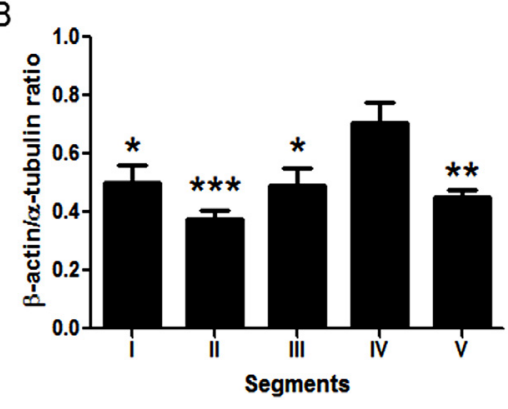

FIGURE 2. (A) Western blots of $\beta$-actin (top) and protein loading control ( $\alpha$-tubulin, bottom) corresponding to segments I through V. Molecular weight markers relative positions are indicated to the right of the membranes. (B) Plot showing mean $( \pm$ SEM) $\beta$-actin to $\alpha$-tubulin ratios from 6 male rats. Asterisks show statistically significant differences with segment IV. ${ }^{\star} P<0.05$, ${ }^{* *} P<0.01$, ${ }^{* * *}$ $P<0.001$; Kruskal-Wallis test.
Fig. 2 shows a representative western blot (A) and quantitation (B). The plot shows the mean ratio $( \pm S E M, n=6)$ of $\beta$-actin to $\alpha$-tubulin. $20 \mu \mathrm{g}$ of total protein were seeded in each lane. Kruskal-Wallis analysis of the data shows statistically significant changes of each segment compared to segment IV ( ${ }^{\star} P<0.05,{ }^{* *} P<0.01$ and $\left.{ }^{* *} P<0.001\right)$. Our results demonstrate that $\beta$-actin levels and distribution changes throughout spermiation. It is also noticeable that its expression peaks immediately after spermiation.

Cell to cell junctions play a key role in the development and formation of the hemato-testicular barrier, which is essential for the full sperm differentiation. Importantly, abnormal expression levels of cytoskeleton proteins (including actin) had been linked to human male infertility (Salvolini et al., 2013). Hence, a better and deeper understanding of the dynamic of $\beta$-actin within these junctions is essential to plan new therapeutic strategies that could be useful to treat pathologies associated with poor or null fertility.

\section{References}

Acosta C, Djouhri L, Watkins R, Berry C, Bromage K, Lawson S (2014). TREK2 expressed selectively in IB4-binding C-fibre Nociceptors hyperpolarizes their membrane potential and limits spontaneous pain. Journal of Neuroscience 34: 1494-1509.

Benitez S, Seltzer A, Acosta C (2017). Nociceptor-like rat dorsal root ganglion neurons express the Angiotensin-II AT2 receptor throughout development. International Journal of Developmental Neuroscience 56: 10-17.

Dym M, Cavicchia J (1977). Further Observations on the BloodTestis Barrier in Monkey. Biology of Reproduction 17: 390-403.

Haskins W, Benitez S, Mercado JM, Acosta C (2017). Cutaneous inflammation regulates THIK1 expression in small C-like nociceptor dorsal root ganglion neurons. Molecular and Cellular Neuroscience 83: 13-26.

Kumar A, Dumasia K, Deshpande S, Gaonkar R, Balasinor N (2016). Actin related protein complex subunit $1 \mathrm{~b}$ controls sperm release, barrier integrity and cell division during adult rat spermatogenesis. Biochimica et Biophysica Acta 1863: 1996-2005.

Li L, Tang EI, Chen H, Lian Q, Ge R, Silvestrini B, Cheng C (2017). Sperm Release at Spermiation Is Regulated by Changes in the Organization of Actin- and Microtubule-Based Cytoskeletons at the Apical Ectoplasmic Specialization-A Study Using the Adjudin Model. Endocrinology 158: 4300-4316.

Losinno A, Lopez L, Capani F, Foscolo M, Ibañez J, Cavicchia J (2012). Beta Actin: Its Implication in the Seminiferous Tubule. In Yoichi Nemoto and Norio Inaba, Testis: Anatomy, Physiology and Pathology. Nova Publishers. New York. 93: 93-111.

Parvinen M. (1993). Cyclic Function of Sertoli Cells. In Russell, L.D., Criswold MD. Chapter XIV, Clerwater FL., The Sertoli Cell. Cache River Press. 14: 331-346.

Parvinen M and Vanha-Pertula T (1972). Identification and enzime quantitation of the stages of the seminiferous epithelial wave in the rat. Anatomical Record 174: 435-450. 
Russell L, Bartke A and Goh J (1989a). Postnatal development of the Sertoli cell barrier, tubular lumen, and cytoskeleton of Sertoli and myoid cells in the rat, and their relationship to tubular fluid secretion and flow. American Journal of Anatomy 184: 179-189.

Russell L, Saxena N, Turner T (1989b). Cytoskeletal involvement in spermiation and sperm transport. Tissue and Cell 21: 361-379.
Salvolini E, Buldreghini E, Lucarini G, Vignini A, Lenzi A, Di Primio R, Balercia G. (2013). Involvement of sperm plasma membrane and cytoskeletal proteins in human male infertility. Fertility and Sterility 99: 697-704.

Tang E, Lee W, Cheng C (2016). Coordination of Actin- and MicrotubuleBased Cytoskeletons Supports Transport of Spermatids and Residual Bodies/Phagosomes During Spermatogenesis in the Rat Testis. Endocrinology 157: 1644-1659. 